\title{
Géolinguistique
}

15 | 2015

La géographie linguistique au Brésil

\section{La contribution d'atlas régionaux pour la cartographie linguistique du portugais du Brésil}

The Contribution of Regional Atlases for the Linguistic Mapping of Brazilian Portuguese

Maranúbia Pereira Barbosa Doiron

\section{OpenEdition}

\section{Journals}

Édition électronique

URL : http://journals.openedition.org/geolinguistique/652

DOI : 10.4000/geolinguistique.652

ISSN : 2650-8176

Éditeur

UGA Éditions/Université Grenoble Alpes

Édition imprimée

Date de publication : 1 décembre 2015

Pagination : 163-171

ISBN : 978-2-84310-317-9

ISSN : 0761-9081

\section{Référence électronique}

Maranúbia Pereira Barbosa Doiron, «La contribution d'atlas régionaux pour la cartographie

linguistique du portugais du Brésil », Géolinguistique [En ligne], 15 | 2015, mis en ligne le 15 février

2019, consulté le 01 novembre 2020. URL : http://journals.openedition.org/geolinguistique/652 ; DOI : https://doi.org/10.4000/geolinguistique.652 


\title{
La contribution d'atlas régionaux pour la cartographie linguistique du portugais du Brésil
}

\author{
Maranúbia Pereira Barbosa Doiron \\ Universidade Estadual de Londrina-CAPES (Brésil), \\ Université Grenoble Alpes
}

\section{Résumé}

La publication des deux premiers volumes de l'Atlas linguistique du Brésil (ALiB , 2014) confirme les avancées des études brésiliennes en dialectologie et géolinguistique et signale la réalisation des recherches dans les disciplines mentionnées au sein de quelques universités du pays. Ces dernières comportent des atlas linguistiques inédits, couvrant toute la surface de quelques États de la fédération brésilienne, ou des aires bien délimitées dans leurs étendues, ou encore des aires linguistiques spécifiques. Beaucoup de ces travaux sont des approfondissements d'atlas déjà publiés, comme l'Atlas Lingüístico do Paraná - ALPR (Aguilera, 1994). Les ouvrages déjà réalisés et ceux qui sont en cours ne représentent qu'un petit exemple de la production de plus en plus abondante dans le monde universitaire consacrée à la réalité linguistique des États brésiliens et de leurs régions. Les résultats, dans l'ensemble, décrivent les variétés dialectales locales et indiquent les différents facteurs qui sont à l'origine du portugais du Brésil.

\section{Mots-clés}

Atlas régionaux, dialectologie, géolinguistique, portugais du Brésil.

\section{Abstract}

The publication of the first two volumes of the Linguistic Atlas of Brazil $(A L i B, 2014)$ confirms the progress of Brazilian studies in Dialectology and Geolinguistics and points to research in the disciplines mentioned 
in some universities of this country. These include unpublished linguistic atlas covering either the entire surface of some states of the Brazilian Federation, or defined areas in their extended, or specific linguistic areas. Many of these works are already published atlases deepening, like Atlas Linguistico do Paraná - ALPR (Aguilera, 1994). The works already realized and others that are underway are only a small example of the production of increasingly abundant in academia dedicated to the linguistic reality of Brazilian states and their regions. The results, overall, describe local dialects and indicate the different factors which have led the Brazilian Portuguese.

\section{Keywords}

Regional atlas, Dialectology, Geolinguistics, Brazilian Portuguese.

\section{Extension territoriale et complexité linguistique}

Dans un pays dont le territoire dépasse les $8500000 \mathrm{~km}^{2}$ on aurait pu s'attendre à ce que la diversité des dialectes existe, sinon dans la même proportion, du moins dans une valeur moyenne égale. Bien que la langue portugaise soit la langue officielle du Brésil, les multiples variétés que l'on rencontre dans toutes ses régions montrent différents éléments qui ont abouti au portugais brésilien tel qu'il est aujourd'hui. Cette délimitation dialectale fait l'objet de nombreuses recherches dans quelques-unes des principales universités du pays, aboutissant à un ensemble d'atlas d'États fédéraux ou de différentes régions qui les composent.

L'urgence d'une cartographie linguistique détaillée du Brésil s'impose dans un scénario dont «la diversité dialectale s'étend partout dans l'espace géographique et social brésilien ${ }^{1} \gg$ (Mattos e Silva, 2004, p. 68). Cette hétérogénéité des dialectes régionaux qui cohabitent au sein d'un complexe réseau socio-culturel où la façon de parler de l'individu, notamment s'il est issu d'une condition économique défavorable, détermine ses limites dans la société. À une telle complexité il faut ajouter aussi le fait que le Brésil est un pays multilingue, bien que les langues des différents groupes ethniques ne soient pas officiellement prises en compte par l'État. Cette réalité linguistique complexe ne passe pas inaperçue chez les spécialistes du portugais du Brésil. Mattos e Silva (2004, p. 71) souligne que dans un pays comme le Brésil, où les grands centres urbains abritent des individus de

1. Texte original : «a diversidade dialetal se espraia por todo o espaço geográfico e social brasileiro». 
différents groupes ethniques, où se côtoient différentes catégories sociales, des migrants ruraux de toutes les régions du pays, une réflexion définitive autour de la norme culte de la langue devient inévitable. Cette dernière apparaît socialement nécessaire pour la manifestation écrite de la langue et aussi pour l'enregistrement formel de la langue orale. Sur ce point, l'auteure rappelle que les dialectes ont leurs propres normes, consensuelles dans la communauté linguistique concernée. Compte tenu de cette diversité, les atlas linguistiques régionaux constituent un important instrument pour la description et l'analyse des phénomènes dialectaux, pour que leurs résultats soient connus principalement par les enseignants et les autres spécialistes de la langue qui pourront contribuer ainsi à la légitimité des variétés dialectales brésiliennes. Dans ce contexte, les atlas linguistiques régionaux de plus petite étendue géographique apportent des informations supplémentaires que les atlas d'État ne peuvent pas relever à cause de leurs réseaux aux mailles plus larges.

\section{Atlas linguistiques d'État et atlas linguistiques régionaux}

Nascentes (1958) est l'auteur de la première division dialectale du Brésil qui rappelle grosso modo la division physico-géographique du pays.

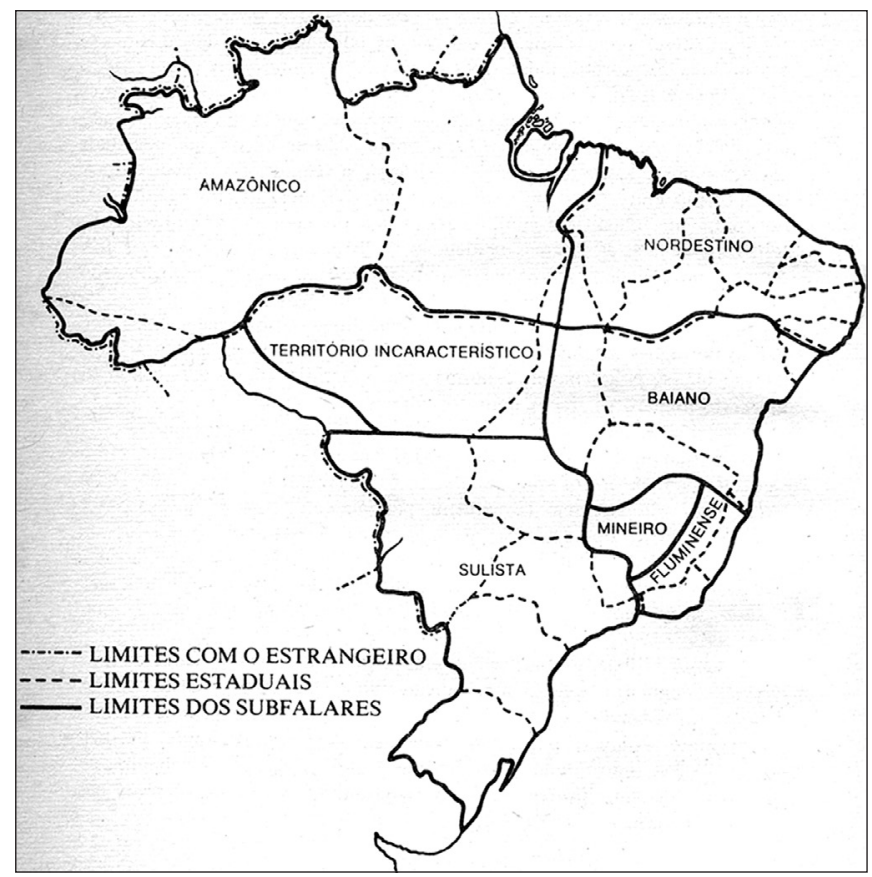

Source : A. Nascentes (1953, p. 18). 
La proposition de Nascentes a été à la base de l'Atlas Prévio dos Falares Baianos - APFB (Rossi et al., 1963), le premier d'une série d'atlas linguistiques d'État qui ont suivi. À l'époque, face à l'impossibilité de produire un atlas couvrant l'ensemble du territoire national, compte tenu des dimensions géographiques, des conditions logistiques déficientes, voire inexistantes, sans compter le manque de ressources financières, la seule alternative plausible a été la réalisation d'atlas d'État, choix qui s'est avéré judicieux et productif. Par ailleurs, c'est justement en travaillant sur les États que les chercheurs peuvent vérifier la pertinence de la division dialectale conçue par Nascentes.

La publication de plusieurs atlas d'États de la fédération brésilienne a encouragé des chercheurs à travailler sur certains faits linguistiques relevés lors de la présentation des données cartographiques, et à envisager la réalisation d'atlas régionaux d'extension aréale plus restreinte. Toutefois, parler d'atlas linguistiques régionaux de petite étendue spatiale, dans un pays de taille continentale tel que le Brésil, peut soulever quelques interrogations sur le concept de dimension géographique. En guise d'information, le premier atlas brésilien, l'APFB, a couvert, seulement dans l'État de Bahia, une surface d'environ $560000 \mathrm{~km}^{2}$, un peu plus grande que les $550000 \mathrm{~km}^{2} \mathrm{de}$ la France constituant la majeure partie de l'ALF (Gilliéron, 1902).

Aguilera (1994), auteure de l'Atlas Lingüístico do Paraná - ALPR, a réalisé des enquêtes dans 65 communes de cet État d'une surface de $200000 \mathrm{~km}^{2}$. Comme dans la plupart des cas, l'auteure n'a pas pu publier toutes les données recueillies auprès de ses informateurs. Altino (2007) a relevé les données qui n'ont pas été analysées dans le cadre de sa thèse de doctorat intitulée Atlas Lingüístico do Paraná II - ALPR II, soutenue à l'université de Londrina. Ce dernier, comme son prédécesseur, contient des cartes sémantico-lexicales, phonético-phonologiques et des cas de unica enregistrés. En s'appuyant sur l'approche dialectométrique, Altino a relevé les indices relatifs de distance et d'identité pour démontrer que la diversité lexicale est associée à la position géographique de cet État et aux aspects spécifiques de sa colonisation.

Ce sont précisément ces derniers qui sont mis en évidence dans la thèse doctorale de Busse (2010) intitulée Um estudo geossociolingüístico da fala do Oeste do Paraná. Dernière à être peuplée, cette région de l'État, vers laquelle ont afflué des colons du sud du pays, principalement à partir des années 1960, se caractérise par un polymorphisme linguistique. Sur un réseau de 9 points, Busse a décrit les dialectes locaux en identifiant des aires homogènes dont les habitants gardent des traces dialectales des premiers colons et des aires hétérogènes où l'on trouve la cohabitation de différents groupes linguistiques dans le même espace. En privilégiant les 
informateurs issus du milieu rural, l'auteure a pu analyser les variations des données recueillies dans les dimensions diatopique, diagénérationnelle, diasexuelle et diastratique. Les données ont été cartographiées, ce qui favorise la visualisation de la dynamique de la variation dans les parlers de l'Ouest du Paraná.

La thèse de doctorat de Cristianini (2007), intitulée Atlas SemânticoLexical da Região do Grande $A B C$, présente une étude dialectale de l'une des plus grandes agglomérations urbaines du Brésil autour de la capitale, São Paulo. Connue sous le nom de Grande $A B C$, la région, formée par 7 villes - Santo André, São Bernardo do Campo, São Caetano do Sul, Diadema, Mauá, Ribeirão Pires et Rio Grande da Serra - , présente un poids considérable dans les contextes socio-politique, économique et culturel de l'État de São Paulo et de l'ensemble du pays. L'importance de la région attire de nombreux habitants des différentes régions du Brésil, ce qui se traduit par une grande mobilité de sa population. En considérant ces caractéristiques, Cristianini a étudié la variation sémantico-lexicale chez cette communauté autour d'un réseau de 9 points, avec l'objectif de conserver leur patrimoine linguistique. L'auteure a utilisé le Questionnaire sémanticolexical de l'ALiB auprès des informateurs des deux sexes, appartenant à deux tranches d'âge - 18 à 30 ans et 50 à 65 ans. L'ouvrage, comportant 202 cartes linguistiques, constitue une référence pour la compréhension des dialectes connus comme paulistas dans la région du Grand $\mathrm{ABC}$. La langue orale dans l'État de São Paulo a fait l'objet d'une autre thèse doctorale portant sur la région littorale : l'Atlas Semântico-Lexical de Caraguatatuba, Ilhabela, São Sebastião et Ubatuba - municípios do Litoral Norte de São Paulo (Encarnação, 2010). Ces deux thèses ont été soutenues à l'université de São Paulo.

Les dialectes désignés comme fluminenses sont le sujet de la thèse Micro Atlas Fonético do Estado do Rio de Janeiro - AFERJ, présentée par Almeida (2008) et dans laquelle sont cartographiées les données de ses investigations auprès de 72 informateurs. L'auteure, qui adopte une approche géolinguistique pluridimensionnelle, a enquêté dans 12 localités de l'État de Rio et a présenté les données relevées dans 307 cartes linguistiques.

La description de quelques localités du littoral de l'État de Rio Grande do Norte a été réalisée par Pereira (2007), auteur de la thèse Atlas Geolingüístico do Litoral Potiguar, sous la direction du professeur Dinah Callou, de l'Université fédérale de Rio de Janeiro.

Silva (2012), auteur de la thèse Atlas Lingüístico do Centro-Oeste Potiguar, a travaillé sur les dialectes parlés dans le territoire de l’État de Rio Grande do Norte, sous la responsabilité du professeur Maria do 
Socorro Aragão, qui a dirigé aussi la thèse Atlas Lingüístico de Pernambuco - AliPE (Sá, 2013).

Augusto (2012) a consacré à l'État de Goiás une thèse intitulée Atlas Semântico-Lexical de Goiás, sous la direction du professeur Irenilde Pereira dos Santos, soutenue à l'université de São Paulo.

Les parlers du Centre-Ouest du Brésil ont fait l'objet de la thèse Atlas Lingüístico e Etnográfico da fronteira Brasil/Paraguai: um registro de línguas em contato, réalisée par Reis (2013). Dans dix localités situées sur la zone de frontière entre l'État de Mato Grosso do Sul et le Paraguay, Reis a pu constater la présence de trois langues, portugais, espagnol et guarani, la dernière étant la langue de la population indienne. Dans cette situation de plurilinguisme se sont développées aussi des variétés dialectales, résultat de la cohabitation des langues en contact.

À propos des parlers sulistas, Romano (2015) a vérifié dans sa thèse si la division dialectale de Nascentes correspond à la réalité linguistique trouvée dans les États qui intègrent cette délimitation. À partir des données de l'ALiB, l'auteur a constaté qu'il n'y a pas une homogénéité absolue dans les parlers sulistas: son travail prouve que la région septentrionale du territoire enquêté présente des différences par rapport à la partie méridionale. Cette thèse met donc en évidence deux grandes aires linguistiques dans un même espace dialectal : l'espace paulista et l'espace sulista. L'influence des dialectes paulistas ne se limite pas à l'État de São Paulo, mais elle s'étend au Nord du Paraná, à l'Ouest de Mato Grosso do Sul, au Sud de Goiás et de Minas Gerais et à la région connue comme Triângulo Mineiro. Quant aux dialectes sulistas, comme a prétendu Nascentes, ils concernent uniquement l'État de Rio Grande do Sul, l'Ouest de Santa Catarina et le Sud-Ouest du Paraná.

\section{Les atlas linguistiques d'État en cours de préparation}

Les atlas d'État qui ont été déjà publiés et, tout récemment l'ALiB, ne diminuent pas l'importance des projets en cours et des nouveaux projets : bien au contraire, ils constituent un encouragement à développer les recherches dialectales au Brésil pour lesquelles se manifeste en ce moment un intérêt accru. Les Brésiliens, y compris ceux qui n'appartiennent pas au monde universitaire sont conscients de l'existence de variétés dialectales qui affleurent dans la langue parlée des différentes régions du pays. Conscients de cette réalité, les linguistes de plusieurs universités brésiliennes encouragent de nouveaux projets de doctorat, certains d'initiative individuelle, dans la mesure où ils contribuent à la connaissance du patri- 
moine linguistique du pays. Nous présentons ci-après quelques-uns de ces projets.

\subsection{Dans la région Nord du Brésil}

- Atlas Etnolingüístico do Acre (ALAC);

- Atlas Lingüístico do Amapá (ALAP);

- Atlas Lingüístico do Mato Grosso (ALMT);

- Atlas Geo-sociolingüístico do Pará : ce projet complète l'Atlas Lingüístico Sonoro do Pará, réalisé par le professeur Abdelhak Rasky, de l'Université fédérale du Pará;

- Atlas Lingüístico de Rondônia (ALiRO).

\subsection{Dans la région Nord-Est}

- Atlas Linguíístico de Alagoas (ALAL) : thèse de la doctorante Maranúbia Pereira Barbosa Doiron, dévéloppée à l'université de Londrina et à l'Université Grenoble Alpes, sous la direction des professeurs Vanderci de Andrade Aguilera et Elisabetta Carpitelli, avec la collaboration de Michel Contini;

- Atlas Lingüístico do Maranhão (ALiMA), sous la coordination générale des professeurs José de Ribamar Mendes Bezerra et Conceição de Maria de Araujo Ramos, tous les deux de l'Université fédérale du Maranhão;

- Atlas Lingüístico do Piauí (ALiPI).

\subsection{Dans la région Sud-Est}

- Atlas Prévio do Espírito Santo (APES);

- Atlas Lingüístico do Estado de São Paulo (ALESP);

- Atlas Lingüístico Sonoro do Estado do Rio de Janeiro (ALiSon-Rio).

\section{Résultats et perspectives}

La liste des thèses de doctorat déjà soutenues et des projets d'atlas d'État cités dans cet article n'est pas exhaustive. En fonction de l'espace limité nous n'avons pas pu les énumérer tous. Le développement des études dialectales au Brésil apparaît dans les nombreuses communications présentées à l'occasion de colloques, symposiums et séminaires organisés par les universités ou d'autres organismes de recherche, brésiliens ou étrangers. Il est visible également dans l'activité d'ouvrages et d'articles dans les revues spécialisées. Nous n'oublierons pas, bien entendu, l'activité des 
dialectologues qui ont été les pionniers de la discipline, toujours présents dans leurs universités : c'est aussi grâce à la continuité de leur action que les études dialectales au Brésil s'inscrivent aujourd'hui dans les orientations plus larges de la linguistique romane.

Actuellement, dans nos universités, les recherches sur le portugais du Brésil commencent à partir de la licence, par des enquêtes limitées le plus souvent à la communauté des étudiants; ultérieurement, au niveau du master et du doctorat, les domaines d'enquête s'étendent à des aires plus vastes, voire même à tout un État. Nous pouvons ainsi envisager que, dans un proche avenir, le territoire brésilien, malgré sa dimension continentale, aura une description de ses principales variétés dialectales, à l'exemple de plusieurs pays européens. Les données de tous les atlas régionaux, d'État et de l'ALiB, permettront de vérifier si la division dialectale proposée par Nascentes est confirmée ou si les frontières des variétés actuelles ne correspondent pas exactement à celles que ce dialectologue avait proposées, comme l'indique apparemment la thèse de Romano (2015). Ce qui ne diminue en rien le mérite de Nascentes, car le fait d'avoir envisagé un classement des variétés dialectales du Brésil dès 1958 a été une initiative notable. Sans oublier qu'avant cette date, en 1923, Nascentes avait déjà publié l'ouvrage $O$ linguajar carioca, consacré aux dialectes de l'État de Rio de Janeiro. Un autre spécialiste, Marroquim, s'est intéressé aux dialectes du Nord-Est du pays, en publiant en 1934 A língua do Nordeste sur ce sujet. En 1944 paraît A linguagem de Goiás, de Teixeira, sur les parlers de cet État. Serafim da Silva Neto, un autre pionnier des études dialectologiques, a publié en 1950 Introdução ao estudo de língua portuguesa no Brasil, en ajoutant plus d'éléments aux études initiales. Leur précurseur, Amaral, avait lui aussi remarqué dès 1920 l'existence d'une variété dialectale dans quelques régions qui comprennent à peu près l'intérieur de l'État de São Paulo, l'Est de l'État de Mato Grosso do Sul, le Sud de l'État de Minas Gerais et le Nord de l'État du Paraná : c'est le dialeto caipira.

La variété dialectale, parallèlement à l'hétérogénéité sociale et économique, est la conséquence d'une histoire qui a connu des épisodes distincts dans toutes les régions du Brésil. Le portugais du Brésil, ni uniforme ni univoque, comme le prétendent quelques puristes, nous le retrouvons dans tous les atlas, ce qui confirme la vision des premiers linguistes. 


\section{RÉFÉRENCES BIBLIOGRAPHIQUES}

Aguilera Vanderci de Andrade, 1994, Atlas Lingüístico do Paraná (ALPR), Curitiba, Imprensa Oficial do Paraná.

Almeida Fabiana da Silva Campos, 2008, Micro Atlas Fonético do Estado do Rio de Janeiro (Micro AFERJ): uma contribuição para o conhecimento dos falares fluminenses, thèse de doctorat, Rio de Janeiro, UFRJ, Faculdade de Letras.

Altino Fabiane Cristina, 2007, Atlas Lingüístico do Paraná (ALPR), vol. II, Londrina, Universidade Estadual de Londrina.

Amaral Amadeu, 1976, O dialeto caipira, São Paulo, Hucitec.

Augusto Vera Lúcia dos Santos, 2012, Atlas Semântico-Lexical de Goiás, thèse de doctorat, São Paulo, Universidade de São Paulo.

Busse Sanimar, 2010, Um estudo geossociolingüístico da fala do Oeste do Paraná, thèse de doctorat, Londrina, Universidade Estadual de Londrina.

Cristianini Adriana Cristina, 2007, Atlas Semântico-Lexical da Região do Grande $A B C$, thèse de doctorat, São Paulo, Universidade Estadual de São Paulo.

EnCARnação Maria Regina Teixeira da, 2010, Atlas Semântico-Lexical de Caraguatatuba, Ilhabela, São Sebastião e Ubatuba - municípios do Litoral Norte de São Paulo, thèse de doctorat, São Paulo, Universidade de São Paulo.

Marroquim Mário, 1945, A língua do Nordeste, São Paulo, Companhia Editora Nacional.

Mattos e Silva Rosa Virgínia, 2004, O português são dois: novas fronteiras, velhos problemas, São Paulo, Parábola Editorial.

NASCENTES Antenor, 1953, O linguajar carioca, Rio de Janeiro, éd. Organizações Simões.

Nascentes Antenor, 1958, Bases para a elaboração de um atlas lingüístico do Brasil, Rio de Janeiro, Casa de Rui Barbosa.

Pereira Maria das Neves, 2007, Atlas Lingüístico do Litoral Potiguar, thèse de doctorat, Rio de Janeiro, Universidade Federal do Rio de Janeiro.

ReIs Regiane Coelho Pereira, 2013, Atlas Lingüístico e Etnográfico da fronteira Brasil/Paraguai, um registro das línguas em contato, thèse de doctorat, Londrina, Universidade Estadual de Londrina.

Romano Valter Pereira, 2015, Em busca de falares a partir de áreas lexicais no Centro-Sul do Brasil, thèse de doctorat, Londrina, Universidade Estadual de Londrina.

Rossi Nelson, Ferreira Carlota \& Isensee Dinah, 1963, Atlas Prévio dos Falares Baianos (APFB), Rio de Janeiro, INL/MEC.

SÁ, Edmilson José de, 2013, Atlas Lingüístico de Pernambuco (ALiPE), thèse de doctorat, João Pessoa, Universidade Federal da Paraíba.

Silva Moisés Batista da, 2012, Atlas Lingüístico do Centro-Oeste Potiguar, thèse de doctorat, Fortaleza, Universidade Federal do Ceará.

Silva Neto Serafim da, 1963, Introdução ao estudo de língua portuguesa no Brasil, Rio de Janeiro, Instituto Nacional do Livro.

TeiXeIra José Aparecido, 1944, Estudos de dialetologia portuguesa: linguagem de Goiás, São Paulo, Anchieta. 